\title{
Análisis
}

\section{Integración regional, soberanía y política exterior en América del Norte}

Introducción

$\mathrm{L}$ a soberanía es la variable clave para una integración norteamericana más profunda, dado que la unificación, tanto económica como social, es ya un hecho palpable. El comercio bilateral de Estados Unidos con Canadá y México pasó de los $\$ 293$ mil millones en 1993 a $\$ 713$ mil millones en 2004. La inversión estadounidense en estos dos países creció con una tendencia similar, de un monto acumulado de $\$ 83$ mil millones a $\$ 210 \mathrm{mil}$ millones. En este momento hay 21 millones de personas de origen mexicano, y más de un millón de canadienses, viviendo en Estados Unidos. Asimismo, alrededor de 700,000 ciudadanos estadounidenses viven en Canadá, y poco más de medio millón en México. Por tanto, no es de sorprender que una encuesta realizada en los tres países en octubre de 2003 señalara que una notable mayoría de personas cree que una unión

* Investigador del Departamento de Estudios del Pacífico, Cucsh-Universidad de Guadalajara y miembro del SNI. económica estadounidense será establecida durante la siguiente década. ${ }^{1}$ Pero, al igual que el búho de Minerva, la integración económica en el continente vuela al atardecer. $\mathrm{El}$ asunto más interesante de la agenda ya no es la formación de una unión económica aún más estrecha entre los tres países, sino los prospectos de una mayor integración y, particularmente, la construcción de una comunidad norteamericana. En los últimos años, y de forma más patente desde los ataques terroristas del 11 de septiembre, cuestiones tales como el ambiente, la migración, la política, la seguridad y el comercio son comúnmente difíciles de separar; el nombre del juego ha cambiado - y su resultado final será determinado en gran medida por un factor: la soberanía.

En el presente artículo me concentro en este atributo y en su relación con el regionalismo, a través de los lentes de la política de reconocimiento e identidad. Mi referente empírico será América del Norte. Más específicamente, indagaré hasta qué punto los asuntos relativos a la soberanía han dictado - y dictarán - la magnitud de la integración regional en el continen- 
te. Procedo de la siguiente forma: en la primera sección abordo la cuestión de la soberanía, dada su relación con la integración regional en América del Norte; en la segunda, discuto la política de identidad y reconocimiento, y en las tercera y cuarta partes, ilustro los retos para una integración regional más profunda en América del Norte mirando, respectivamente, las tradiciones de la política exterior de México y Canadá, y hago hincapié en la manera en que han tratado con su vecino común. Concluyo con una breve reflexión sobre los problemas que se avecinan.

\section{La soberanía importa}

Tal como lo presentó un reporte del Woodrow Wilson International Center for Scholars en el año 2002, la manera en que "los ciudadanos de Estados Unidos, México y Canadá definan [la] identidad y soberanía" de sus países, determinará hasta qué punto emergerá una comunidad de América del Norte. ${ }^{2}$ La soberanía es crucial porque encapsula las concepciones que cada país tiene de su propia identidad e intereses nacionales. Por ejemplo, se puede argumentar que para Estados Unidos la defensa de su sistema constitucional ha figurado en un lugar importante entre sus principales intereses nacionales porque coincide con su identidad básica; cualquier acuerdo internacional que se perciba como contrario a éste probablemente será rechazado con el argumento de la soberanía. La soberanía es el cristal a través del cual son percibidos los proyectos de cooperación e integración. Es por ello que en el contexto norteamericano, si el permitir la inversión privada en la extracción de petróleo (México), o la apertura de las industrias culturales (Canadá) o la legalización de trabajadores indocumentados (Estados
Unidos), son considerados contrarios a la soberanía del país, el proyecto estará condenado al fracaso.

Pero la soberanía no es ni inmutable ni meramente idiosincrática. Lo que cada país considera que está entre sus intereses nacionales cambia con el tiempo. Promover un orden internacional liberal, por ejemplo, no era tan importante para Estados Unidos antes de la Segunda Guerra Mundial como lo fue durante la Guerra Fría. Además, lo que el interés nacional significa para cada Estado está determinado, en parte, por las demandas y el reconocimiento de los otros: la soberanía es, entonces, un atributo concedido a los estados por la comunidad internacional, así como la igualdad de ciudadanía era conferida a los individuos por la polis; es un concepto inherentemente relacional. Es por ello que mientras Canadá, México y Estados Unidos mantengan concepciones de soberanía que permitan exclusivamente la integración económica, el surgimiento de una comunidad norteamericana no se verá materializado. Ciertamente existen algunos efectos de derrame desde la integración económica, pero la construcción de una comunidad no es uno de ellos. Por tanto, la soberanía es más -y no menos- importante en la economía política global posterior al 11 de septiembre; lejos de ser obsoleta, como lo sugieren algunos comentaristas, todavía define el contorno de las aspiraciones e identidades nacionales. ${ }^{3}$

Resulta interesante que las innumerables propuestas, artículos y reportes publicados recientemente acerca de la integración norteamericana difícilmente mencionan el asunto de la soberanía. He aquí dos ejemplos prominentes: Security and Prosperity Partnership of North America, preparado por ministros estadounidenses designados por sus líderes para producir 
un reporte en la Cumbre de Marzo de 2005, y Building a North American Communi$t y$, el reporte de un cuerpo independiente de trabajo patrocinado por el Council on Foreign Relations en asociación con el Canadian Council of Chief Executives y el Consejo Mexicano de Asuntos Internacionales. El primer documento ni siquiera menciona la soberanía, está limitado a materias de competitividad y seguridad. Hablar de soberanía parece ser tabú en el actual proceso de integración dirigido por el Estado. El segundo reporte menciona la soberanía tres veces - pero principalmente para reiterar que debería permanecer tal y como está-. Aunque el esfuerzo no-gubernamental es más ambicioso que el inter-gubernamental, sugiriendo una serie de tópicos y pasos que llevarían al avance en la creación de una comunidad norteamericana, el mecanismo clave para alcanzar las metas lo elude. Está enmarcado simplemente en los términos de "seguridad, prosperidad y oportunidad". 4 Enfrentémoslo: la creación de una comunidad norteamericana implica la redefinición de la soberanía de tres países.

\section{La política del reconocimiento}

Históricamente, las cuestiones de soberanía han estado en el corazón de las relaciones norteamericanas; y aunque su transformación parcial es lo que ha permitido el actual estado de integración, si la concepción de soberanía de cada Estado no se ve transformada e imbuida de un nuevo significado, la creación de una comunidad norteamericana continuará siendo una quimera. Sencillamente no sucederá. Incluso si una comunidad viable en América del Norte no requiriera el compartir la soberanía, en algunas áreas, como ha sucedido en la Unión Europea, para poder avanzar sería necesaria una reconceptuación sustancial de este atributo fundamental. Por ello, el reconocimiento de los distintos valores nacionales es fundamental en el proceso amplio de integración continental.

La historia de los dos socios más pequeños en América del Norte ilustra claramente lo importante que resulta el factor del reconocimiento. Canadá y México han sido profundamente afectados por su interacción con Estados Unidos; su vecino común ha forjado de forma significativa su identidad y sus intereses. Como lo nota Herschel Hardin, "Si Estados Unidos no existiera, tampoco Canadá" existiría. El temor de México por las ambiciones anexionistas de su vecino ha existido prácticamente desde su establecimiento como un país independiente - eso es, antes de la Guerra de 1846-1848 en la que perdió la mitad de su territorio-. ${ }^{6}$ Yo señalaría que, en el fondo, las relaciones bilaterales de Canadá y México con su contraparte han sido una batalla para ganar reconocimiento. Estados Unidos históricamente ha sido, y continuará siendo en el futuro inmediato, el mayor poder en América del Norte. Para Canadá, el miedo de perder sus diversos mercados culturales y sus características nacionales en la interacción con Estados Unidos ha sido una constante, mientras que para México la pérdida de su integridad territorial en manos de su vecino del Norte, acompañada de la percepción estadounidense de México como su patio trasero, ha producido un sentimiento de soberanía definido por sus temores acerca de Estados Unidos.

La lucha por el reconocimiento es una parte integral de la soberanía - y no sólo en América del Norte, como lo ilustra claramente el repetido intento de Rusia por ser considerado un país europeo- ${ }^{7}$ 
El reconocimiento es un acto social que otorga a las diferencias un significado particular, a la vez que constituye a un actor como sujeto. Es, en este sentido, como lo señala Alexander Wendt, que "la subjetividad depende de la inter-subjetividad". ${ }^{8}$ Lo que creemos ser depende parcialmente de cómo nos perciben los demás. Pero los agentes, como Hegel notó hace mucho tiempo, se esfuerzan por ser reconocidos, no por conceder reconocimiento. De ahí la lucha por conseguirlo. Además, el reconocimiento no es un concepto lineal. Hay al menos dos diferentes niveles de reconocimiento, negativo y positivo, o delgado y grueso. El primero implica ser reconocido como persona independiente dentro de una comunidad legal: en el caso del sistema internacional, ser reconocido como estado soberano. Este tipo de reconocimiento es lo que hace a

El asunto más interesante de la agenda ya no es la formación de una unión económica aún más estrecha entre los tres países, sino los prospectos de una mayor integración $\mathrm{y}$, particularmente, la construcción de una comunidad norteamericana los actores formalmente iguales. Sin embargo, el reconocimiento grueso, también requiere que lo que sea que hace único al individuo (o al Estado) sea considerado como valioso por sí mismo. Es este segundo tipo, el reconocimiento positivo o grueso, el que resulta especialmente relevante para el potencial proceso de construcción de una comunidad en América del Norte.

De manera significativa, algunos aspectos de la tradición liberal que han dado forma a los debates políticos en Canadá, México y Estados Unidos, tocan este tema. ${ }^{9}$ Más allá de los diferentes acentos nacionales en materias como el rol del Estado, la comunidad, y el individuo, esta postura compartida habla de la necesidad de reconocer las diferencias culturales que implicaría la creación de una comunidad norteamericana. La razón por la que afirmo que una integración más profunda del continente requeriría tratar abiertamente las diferencias nacionales es doble: primero, porque sólo cuando existe un reconocimiento recíproco emerge un sentido de identidad colectiva, y segundo, porque sólo entonces el individuo (en este caso, el Estado) siente la suficiente confianza como para sumarse a un proyecto que lleve a la reconceptuación de sí mismo (en este caso, la soberanía). Por ejemplo, si el actor A sabe que sus particularidades son genuinamente reconocidas por B y C, este conocimiento probablemente tendrá el efecto de lograr que A se sienta menos amenazado por el ambiente externo, y también -lo que es más importante para mi argumento-, de actualizar las potencialidades de A. Ya que A sabrá mejor cómo actuar en este ambiente más seguro, será un agente más completo. ${ }^{10}$ Como Ringmar ha notado:

\footnotetext{
Desear reconocimiento no es desear un objeto que provea de utilidad, placer o beneficio, sino desear ser un sujeto de naturaleza determinada. El deseo de reconocimiento es lo fundamental del deseo humano. ${ }^{11}$
}

Es por ello que los Estados-nación -incluyendo aquellos que se embarcan en procesos de integración — no se despojan de su identidad simplemente; desean que sus rasgos distintivos sean reconocidos por los otros. ${ }^{12}$ 
Como lo señala Samuel Huntington al referirse a los países pos-comunistas, "Las personas pueden aspirar a una identidad pero no ser capaces de lograrla, a menos que sean bienvenidas por aquellas que ya poseen esa identidad. El factor crucial de la pos-Guerra Fría para las personas del Este de Europa era si el Oeste aceptaría que se identificaran a sí mismas como parte del Oeste". ${ }^{13}$ En el escenario norteamericano esto probablemente significaría —más que un crisol o un enfoque mestizo de la integración - un mosaico al estilo del canadiense, en el que, a pesar de la convergencia regional, las diferencias nacionales fueran adoptadas abiertamente. ${ }^{14}$

En este proceso, las decisiones de política exterior de los tres estados serán cruciales. Como Stephanie Golob afirma, la política exterior

provee de tres cosas a los ciudadanos de una nación: soberanía, seguridad e identidad. La soberanía dicta que el gobierno y los ciudadanos del Estado ("nosotros") decidan las políticas, la identidad define 'quiénes somos' como nación, y la seguridad protege la soberanía y la identidad de la nación. Los gobiernos deben convencer a los ciudadanos de que los proyectos regionales son consistentes con estos tres valores, mediante la expansión de la definición del "nosotros". ${ }^{15}$

\section{Soberanía, seguridad e identidad de México}

Como se ha mencionado, la vida de México como país independiente ha estado profundamente influida por su vecino del Norte. Pero la influencia no se ha limitado a la pérdida territorial del país; el campo de la ideología también ha sido importante. De esta manera, por ejemplo, desde los albores de la República las nuevas y progresistas ideas políticas de Estados Unidos causaron un profundo efecto en México. El resul- tado fue, por supuesto, una Constitución creada con base en la que fuera elaborada por los padres fundadores de Estados Unidos - para un contexto socio-político muy distinto, obviamente-. Es por esta razón que, en el Congreso Constituyente de 1823, Servando Teresa de Mier se oponía a fundamentar la Constitución Mexicana en la de su vecino del Norte. "La nación," advirtió, "careciendo de un programa, aceptó ilusoriamente uno ya elaborado que era de manufactura norteamericana... la consecuencia ha sido que ahora todo en México tiene la marca de fábrica de ese origen". ${ }^{16}$ A partir de ese episodio, escribió Edmundo O'Gorman, "México entra al sendero de la adaptación en lugar de al camino de la libertad". ${ }^{17}$

Entonces, desde su vida temprana como país independiente, México albergó sentimientos encontrados, ambivalentes, respecto a su vecino del Norte: el epítome de la democracia era también un peligro expansionista. Es por ello que la guerra de 1846-1848 fue tan devastadora para la causa liberal. Después de que la amenaza se materializara, la percepción negativa de Estados Unidos imperó en México. La concepción maximalista de soberanía que surgió, una que veía como interferencia en las cuestiones internas cualquier discusión acerca de asuntos internos mexicanos en el exterior, se rodeó de un profundo sentimiento anti-estadounidense. A partir de finales de la década de 1840, Estados Unidos se convirtió en un peligro permanente para la seguridad de México. Pero, como con cualquier elemento formativo reprimido posteriormente, el rol ejemplar de ese país ha salido a la superficie reiteradamente conjurando a México. La influencia que tuvo el primero en este último, y la afinidad legítima entre programas políticos de algunos grupos a ambos lados 
del Río Grande/Río Bravo, no pueden ser simplemente descartadas. Así, como afirmara Octavio Paz, "Los Estados Unidos son la negación de lo que nosotros éramos en los siglos dieciséis, diecisiete y dieciocho, y de lo que, desde el siglo diecinueve, muchos entre nosotros preferiríamos ser". ${ }^{18}$ La identidad del Estado mexicano ha sido entonces, en gran medida, conformada por su vecino del Norte.

De tal forma, no sorprende que México tradicionalmente haya sido uno de los más fieles partidarios del principio de no-intervención en el hemisferio. Al respecto, resulta ilustrativa la Doctrina Estrada de 1930, que sostiene que México no se pronuncia con respecto a otorgar o negar el reconocimiento de otro gobierno, porque esa práctica "lastima la soberanía de las naciones" a cuyo régimen se está concediendo (o rehusando) el reconocimiento. Así, por ejemplo, México no cortó las relaciones diplomáticas con la Cuba castrista. Además, desde 1988 el principio de no-intervención en la conducta de la política exterior mexicana está consagrado en la Constitución. ${ }^{19}$ De esta forma, la política exterior ha estado en el centro de la identidad del país.

La autonomía de la política exterior mexicana (respecto a la estadounidense) siempre ha sido relativa, por supuesto. México alcanzó un acuerdo tácito con su vecino del Norte acerca del "derecho al desacuerdo" en asuntos secundarios. Como Bernardo Mabire señala: "Por un largo tiempo el gobierno de México fingió en su retórica cierto radicalismo de izquierda, y el de Estados Unidos amablemente pretendió que le creía". ${ }^{20}$ Por ello, el presidente mexicano Gustavo Díaz Ordaz afirmó a Lyndon B. Johnson en 1964 que "habrá una ventaja considerable [para ambas naciones] si México es capaz de continuar demostrando su independencia política, no estando de acuerdo con Estados Unidos en cuestiones relativamente secundarias". ${ }^{21}$ Así, durante la crisis de los misiles de 1962, México se sumó decididamente a las acciones de la administración Kennedy, argumentando que la presencia de misiles soviéticos en Cuba constituía "una amenaza a la paz y seguridad del hemisferio". ${ }^{22}$

De esa manera, el tercermundismo de Luis Echeverría durante la década de 1970, y la política activista hacia América Central de López Portillo a finales de los años setenta y principios de los ochenta, fueron tolerados por Washington. Esta independencia era una valiosa posesión que servía para legitimar domésticamente el régimen pos-revolucionario. Como Jorge G. Castañeda, un firme crítico del régimen mencionado, reconoció: "el México moderno posee una virtud redentora: una política exterior propia". ${ }^{23}$ El convenio bilateral también implicó que México estaría relativamente aislado de las presiones estadounidenses sobre derechos humanos y cuestiones de democracia. ${ }^{24}$ Como Robert Pastor ha sugerido, "no hay país en el mundo que sea más sensible que México a los esfuerzos por influirlo de Estados Unidos, y ningún país tan exitoso en resistir la influencia estadounidense". ${ }^{25}$

Por tanto, hasta mediados de la década de 1980 la política exterior de México correspondió con su modelo nacionalista de desarrollo (el de sustitución de importaciones), que era considerado por algunos autores como su ancla. ${ }^{26} \mathrm{Y}$ como la crisis de la deuda en 1982 hizo evidente el agotamiento de ese modelo de desarrollo, el marco de política exterior de México también se percibía como manifiestamente inadecuado. ${ }^{27}$ Fue hasta algunos años después, a medida que la administración de Carlos Salinas (1988-1994) intensificara el 
proceso de reforma estructural y la liberalización comercial iniciada por su predecesor (por ejemplo, México se había embarcado en un amplio programa de privatización y se convirtió en miembro del GATT, Acuerdo General sobre Aranceles y Comercio) que la política exterior mexicana comenzó a cambiar de manera más pronunciada. El factor principal fue, claramente, la contraparte del país: Estados Unidos. En consecuencia, Salinas decidió negociar en 1990 un tratado de libre comercio con ese país. ${ }^{28} \mathrm{Su}$ administración consideró que México no tenía más opción que establecer una relación más cercana con su vecino del Norte. ${ }^{29}$ Como Susan Kaufman ha observado: "Este cambio en la estrategia de desarrollo ha llevado a México a remarcar las oportunidades inherentes de una relación más cercana con Estados Unidos, en lugar de exagerar los costos potenciales". ${ }^{30}$

Por consiguiente, la naturaleza de la política exterior de México estaba tomando un nuevo giro a principios de 1990. Jorge Chabat afirma que la decisión de Salinas de incluir el desarrollo económico como un objetivo explícito de la política exterior, constituía "una redefinición radical del entendimiento tradicional de México de la soberanía nacional, que tradicionalmente sólo había tenido un eje doméstico" ${ }^{31}$ Esta reconceptuación forma parte de lo que él llama "la 'economización' de la política exterior". ${ }^{32}$ Castañeda coincide:

No es una exageración afirmar que el presidente Carlos Salinas de Gortari ha reducido las relaciones internacionales de México a relaciones económicas internacionales, y que éstas, a su vez, se han reducido casi completamente a la dimensión estadounidense. ${ }^{33}$

La economización de las relaciones de México con Estados Unidos, o más precisamente, el TLCAN, se ha convertido en la "nueva gran estrategia" mexicana de la
pos-Guerra Fría. ${ }^{34}$ Éste no es un hecho inesperado, dadas las circunstancias. Como Castañeda ha observado:

\begin{abstract}
No tenía sentido, por un lado, poner todos los huevos de México en una canasta (a saber, aquella que contenía financiamiento externo, seguridad en los negocios y el apoyo estadounidense) y entonces proceder a quejarse y discutir con los dueños de la canasta. ${ }^{35}$
\end{abstract}

Así, durante la administración de Salinas, la finalidad de la política exterior de México era establecer lazos aún más cercanos con Estados Unidos. ${ }^{36}$ Ya se habían esfumado los esfuerzos mexicanos de principios de los ochenta por actuar como un poder independiente de medianas dimensiones. Como Humberto Garza Elizondo nota: "Muy pocas cosas cambiaron más durante el sexenio de Salinas que la política exterior". ${ }^{37}$ Los asuntos económicos vinieron a ocupar el lugar principal en la relación de México con su vecino, como se evidenció con el reemplazo de la Secretaría de Relaciones Exteriores por la de Comercio en la conducción de las relaciones bilaterales. ${ }^{38}$

\section{Soberanía, seguridad e identidad de Canadá}

De manera similar, el espectro de Estados Unidos se ha proyectado considerablemente sobre Canadá. La misma fundación de Estados Unidos de América dejó una marca indeleble en su vecino del Norte, convirtiéndolo en una leal fortaleza del imperio Británico. Casi un siglo después, cuando la Confederación Canadiense había sido establecida, Thomas D'Arcy McGee, uno de sus arquitectos, reconocería que: "A diferencia de nuestros vecinos [los padres de la Constitución estadounidense] nosotros no teníamos cuestiones que resolver respecto a la soberanía". ${ }^{39}$ Este sentimiento de pertenencia —al menos en la parte británica 
de Canadá-a un poder extra continental, ciertamente mantuvo distanciados por varias décadas a los dos países situados en el extremo norte del hemisferio occidental.

Pero también estaban sus innumerables características en común. Como lo expusiera Seymour Lipset, "[Estados Unidos y Canadá] se parecen más entre ellos que cualquier otro par de naciones". ${ }^{40}$ Y, como Lawrence Taylor ha observado: "Aunque oficialmente se considera a los canadienses como extranjeros en Estados Unidos, socialmente no son vistos como tales". ${ }^{41}$ Esto, por supuesto, no significa que los canadienses carezcan de una identidad distintiva. Para empezar, Canadá es una sociedad mucho más moderada y orientada hacia la comunidad que Estados Unidos. Sus programas de desarrollo regional y su ampliamente aclamada política universal de servicios médicos son sólo dos muestras de la importancia que otorga Canadá a la igualdad social. Pero, generalmente, los canadienses también son conservadores, solemnes y respetuosos de la autoridad. ${ }^{42}$ Como Lipset notara hace más de 40 años, "Canadá ha sido y continúa siendo más clasista, elitista, respetuosa de la ley, estatista, orientada hacia la comunidad y particularista que Estados Unidos". ${ }^{43}$

Sin embargo, con frecuencia esta identidad ha sido definida principalmente por su contraparte (Estados Unidos). Como Taylor señala: "Se puede decir [...] que los canadienses son los 'no-estadunidenses' más antiguos del mundo, y que, de hecho, sin cierto espíritu antiamericanista Canadá no podría existir". ${ }^{44}$ Es por ello que la identidad se ha convertido en la "quintaesencia de los asuntos canadienses". ${ }^{45}$ Temiendo la hegemonía cultural estadounidense, los canadienses luchan por establecer su propia identidad distintiva. Sus similitudes sociales con su mucho más poderoso y poblado vecino son una advertencia constante de los riesgos de la asimilación cultural. En este sentido, la postura de Canadá contrasta con la mexicana. Así pues, los "canadienses, más que los mexicanos, se preocupan por la 'pérdida' de identidad". ${ }^{46}$

A causa de ello, históricamente la política exterior canadiense ha tratado de mantener una distancia crítica con Estados Unidos. Incluso, aunque los dos países pertenecen a la misma alianza de seguridad (OTAN) formada bajo la hegemonía estadounidense luego de la Segunda Guerra Mundial, y podría decirse que forman una "comunidad de seguridad", tradicionalmente Canadá ha permanecido ajena a las aventuras militaristas y a los impulsos más unilaterales de política exterior de su vecino del Sur — como su política hacia Cuba-. Simultáneamente, Canadá ha intentado desarrollarse en la línea de un modelo económico más intervencionista y nacionalista que el de Estados Unidos, con mayor regulación del mercado y una política industrial propia. Como en el caso mexicano, esto por supuesto no representa más que una autonomía relativa con respecto a Estados Unidos; factor primordial históricamente, este último, en la política exterior general de Canadá. ${ }^{47}$

Pero, una vez más como en el caso mexicano, a principios de la década de 1980 el nacionalista modelo económico y diplomático canadiense comenzaba a decaer. Por tanto, en 1983 una profunda revisión de la política comercial, llevada a cabo por el gobierno de Pierre Trudeau, sugirió la negociación de un tratado de libre comercio sectorial con Estados Unidos. Cuando el gobierno conservador del primer ministro Brian Mulroney subió al poder al año siguiente, la estrategia de liberalización cobró impulso, materializándose cinco 
años después, cuando el Tratado de Libre Comercio Canadá-Estados Unidos entró en vigor. ${ }^{48}$ Así, para la última década del siglo veinte, tanto Canadá como México habían cambiado formalmente el rumbo de sus tradiciones de política exterior, acercándose a su vecino común (vale la pena recordar que en 1979, cuando el presidente Ronald Reagan propuso a López Portillo y Trudeau la negociación de un tratado de libre comercio, éstos declinaron la invitación). ${ }^{49}$

\section{Lo que está por venir}

Una década después de la entrada en vigor del TLCAN, es claro que la integración económica no es el asunto principal en América del Norte - eso es ya un hecho. Ahora la cuestión principal es cómo dirigir los asuntos relativos a ésta, desde el ambiental hasta el migratorio, pasando por la disparidad económica entre México y sus dos socios, que sólo un nuevo entendimiento del proceso de regionalización puede tratar de forma adecuada. La mera búsqueda de los nobles principios de no-intervención y autodeterminación ya no es una opción viable para Canadá y México. Igualmente, es tiempo de que Estados Unidos reconozca las inquietudes relativas a la identidad y la seguridad de sus vecinos, y de que haga movimientos sustantivos que puedan llevar adelante la nueva agenda común.

A finales de la década de los años noventa, durante los gobiernos de Chrétien, Clinton y Zedillo, sus tres ministros de relaciones exteriores se reunieron en Santiago de Chile en el marco de la Cumbre de la Organización de Estados Americanos, y acordaron lanzar una agenda trilateral que fuera más allá del comercio. Casi al mismo tiempo, una encuesta llevada a cabo en los tres países encontró que una clara mayoría de la población estaba en favor de una integración política más estrecha, siempre y cuando se cumpliera con al menos una de las dos condiciones siguientes: 1. Mejor calidad de vida, 2. Solución más efectiva a la cuestión ambiental. ${ }^{50}$ Por tanto, en esta era globalizada, el contexto norteamericano podría ser un laboratorio interesante para descubrir cómo el proceso de regionalización afecta uno de los todavía centrales principios del sistema internacional: la Soberanía. nखy

\section{Notas}

1. Datos de Pastor 2005, 2-3, 7, NewsMax.com Domingo, julio 3, 2005, y shelteroffshore.com.

2. Heard, Emily. 2002. Toward a North American Community (Washington, Woodrow Wilson Center), 32.

3. Haass, Richard. 2005. "Sovereignty". Foreign Policy Sep/Oct 150: 34.

4. De forma similar, un artículo reciente sobre el décimo aniversario del TLCAN menciona a la soberanía, brevemente y una sola vez. Véase: Ordorica, Ana Paula. 2005. "Una década del TLCAN y sus impactos." Foreign Affairs en Español (5) 3.

5. En Gwyn, Richard. 1985. The 49th Paradox: Canada in North America (Toronto, McClelland and Stewart), 195.

6. Taylor Hansen, L. D. 2001. El Nuevo Norteamericano. México, Universidad Nacional Autónoma de México: 63-4.

7. Ringmar, Erik. 2002. "The Recognition Game: Soviet Russia Against the West." Cooperation and Conflict (37) 2: 115-136.

8. Wendt, Alexander. 2003. "Why a World State is Inevitable?" European Journal of International Relations 9 (4), 511.

9. Como Robert Earle y John Wirth ha notado, los caminos convergentes en que las sociedades Norteamericanas se han embarcado se nutren de un patrimonio liberal común. Cf. Earle, Robert, y John Wirth. 1995. "Conclusion: The search for Community." Identities in North America. R. L. Earle y J. D. Wirth. Stanford, California, Stanford University Press: 205.

10. Möller, Ulrika. 2004. "Multilateral Negotiations: A Security or a Recognition Game? In Search of an Explanation for Why Key-Players Opt Out." Ensayo presentado en la Quinta Conferencia Pan-Europea de Relaciones Inter- 
nacionales: Construyendo Órdenes Mundiales en La Haya, Septiembre 9, 29.

11. Ringmar op. cit.: 119.

12. Wendt op. cit.: 515 .

13. Huntington, Samuel. 2004. Who Are We? The Challenges to America's National Identity (Simon \& Schuster): 23.

14. Como Inglehart et al 1996 ha señalado: “... nuestra evidencia indica que la tendencia a largo plazo en los tres países se dirige a una perspectiva global en aumento. Un nacionalismo estrecho que había sido dominante desde el siglo diecinueve está, gradualmente, dando paso a un sentido más cosmopolita de la identidad (1)" ... “.. los sistemas de valores de las tres sociedades Norteamericanas están cambiando gradualmente y la dirección del cambio de valores es consistente con las amplias transformaciones que están tomando lugar en los estados más industrializados. Los Norteamericanos se encuentran en una trayectoria común de cambio que se dirige a

1. economías de libre mercado

2. instituciones políticas democráticas

3. globalización.” Inglehart, Ronald, et al. 1996.

The North American Trajectory. New York, Aldine de Gruyter: 23.

Además, "A través de las tres naciones, los sentimientos de pertenencia a una unidad geográfica mayor a la nación fueron de una escala promedio de 9 \% en 1981 a 17 \% en 1990 (161)." De cualquier forma, como Ruy Sánchez (en Earle y Wirth 1995: 55) ha notado, “¿Es posible construir una nueva concepción de identidad que también asuma nuestra otredad natural, o naturaleza multinacional?... Nuestro reto es compartir nuestras diferencias y nuestras identidades nacionales". En Earle, R. L. and J. D. Wirth. 1995. "Introduction." Identities in North America. R. L. Earle and J. D. Wirth. Stanford, California, Stanford University Press.

15. Heard, Emily. 2002. Toward a North American Community? A Conference Report (Washington, Woodrow Wilson International Center for Scholars): 10 .

16. En Valdes-Ugalde, Francisco. (2004). "Janus and the Northern Colossus: Perceptions of the United States in the building of the Mexican Nation." The Journal of American History 86 (2): 50.

17. Ibid.

18. Valdes-Ugalde, op. cit.: 1.

19. En el Artículo 89.

20. Mabire, Bernardo. 1994. "El fantasma de la antigua ideología y su resistencia al cambio de la política exterior en el sexenio de Salinas de Gortari." Foro Internacional 34(4): 545.

21. Citado en Aguayo Quezada, Sergio. 1998. El panteón de los mitos: Estados Unidos y el nacionalismo mexicano. México, Grijalbo-El Colegio de México: 110.

vol. 10, núm. 28 / enero-abril de 2007
22. Ibid., 111.

23. Castañeda, Jorge. 1990. "Salinas's International Relations Gamble." Journal of International Affairs 43(2): 407.

24. Serrano Carreto, M. (1996). "La herencia del cambio gradual. Reglas e instituciones bajo Salinas." Foro Internacional 35(1-2): 446.

25. Pastor, Robert, y Jorge Castañeda. 1989. Limits to Friendship: The United States and Mexico. New York, Vintage Books: 10.

26. Chabat, Jorge. 1997. "Mexico's Foreign Policy after NAFTA: The Tools of Interdependence," en Rodolfo O. de la Garza and Jesús Velasco (eds). Bridging the Border: Transforming Mexico-U.S. Relations. Lanham: Rowman \& Littlefield: 35.

27. Véase Garza Elizondo, Humberto. 1994. "Los cambios de la política exterior de México: 1989 1994." Foro Internacional. (34) 4: 534-544.

28. La propuesta original de México no incluía a Canadá.

29. Véase: Mabire, op. cit.

30. Kaufman, Susan. 1990. "U.S.-Mexico Relations: An Optimistic View." Journal of International Affairs. 43 (2), pp. 424.

31. Chabat, op. cit: 37.

32. Chabat, Jorge. 1991. "Mexico's Foreign Policy in 1990: Electoral Sovereignty and Integration with the United States." Journal of Interamerican Studies and World Affairs. 33 (4): 2.

33. Castañeda, op. cit.: 408.

34. Mares, David R. 1996. "Strategic Interests in the U.S.-Mexican Relationship," en John Bailey and Sergio Aguayo Quezada (eds.). Strategy and Security in U.S.-Mexican Relations beyond the Cold War. San Diego: University of California: 36.

35. Castañeda, op. cit: 410.

36. Mabire op. cit.: 545.

37. Garza Elizondo, op. cit: 536.

38. Chabat 1991, cit.: 2-5.

39. Lipset, Samuel. 1990. The Values and Institutions of the United States and Canada. New York, Canadian American Committee: 43.

40. En Lipset, op. cit.: 212.

41. Taylor, op. cit.: 115-6.

42. Pierre Breton en Lipset, op. cit.: 44.

43. En Inglehart, op. cit: 12.

44. Taylor, op. cit.: 128 .

45. Lipset, op. cit.: 42.

46. Earle y Wirth 1995: 5.

47. McKenna, P. (1999). "Canada, the United States, and the Organization of American States." The American Review of Canadian Studies 29(3): 474.

48. Barry, Donald. 1995. "The Road to NAFTA," en Barry, D. (ed.). Toward a North American Community? Canada, the UNited States, and Mexico. Boulder, Westview Press: 7.

49. Ibid.: 5 .

50. Inglehart, op. cit.: 145 . 